\title{
Left ventricular outflow tract
} obstruction following an uncomplicated primary percutaneous coronary intervention: a recognized but rare

European Heart Journal: Acute Cardiovascular Care 2(I) 68-7I

(C) The European Society of Cardiology 2012

Reprints and permission:

\author{
S Khan, DP Ripley, MA de Belder, AT Goodwin, N Barham and \\ RA Wright
}

\begin{abstract}
Dynamic left ventricular outflow tract obstruction is a rare but important complication of myocardial infarction. It occurs acutely and may mimic the presentation of papillary muscle rupture or acquired ventricular septal defect. Unlike these mechanical complications, it does not require circulatory support or cardiac surgical intervention. Recognition is critical because it typically responds to volume loading and beta blockade. We report a case who displayed many classical features of this condition.
\end{abstract}

\section{Keywords}

Left ventricular outflow tract obstruction, LVOT obstruction, primary percutaneous coronary intervention, severe mitral regurgitation, acute myocardial infarction

Received: I3 July 2012; accepted: 25 November 2012

\section{Introduction}

Dynamic left ventricular outflow tract (LVOT) obstruction associated with systolic motion of the anterior mitral leaflet has become a recognized complication in patients with acute myocardial infarction. ${ }^{1-3}$ Little is known about the incidence and predictors of this phenomenon in patients without hypertrophic obstructive cardiomyopathy or other congenital lesions. An increased LVOT pressure gradient associated with hypotension can occur after acute myocardial infarction due to distortion of left ventricular geometry and increased contractility of the preserved myocardial segments. We report the development of dynamic LVOT obstruction after an uncomplicated angioplasty for anterior ST-elevation myocardial infarction, leading to hypotension and cardiogenic shock. This complication has become increasingly recognized although a full understanding of the pathogenesis has not been established.

\section{Case report}

A 73-year-old man presented directly to the cardiac catheterization laboratory with a history of ischaemic chest pain and evidence of anterior ST-elevation on his ECG. On arrival he was in sinus rhythm with normal heart sounds and no evidence of heart failure. There was no significant past medical history.

Immediate angiography performed via a right radial approach showed minor disease in the left main stem artery. The left anterior descending artery had diffuse disease with a severe mid-vessel bifurcation stenosis. The circumflex was also diffusely diseased with a severe lesion in its midpart. The dominant right coronary artery showed mild-tomoderate disease at the origin of the posterior descending branch. Immediate angioplasty of the left anterior descending artery with deployment of a drug-eluting stent and balloon angioplasty of the diagonal branch was performed. The circumflex artery lesion was not treated.

The James Cook University Hospital, Middlesbrough, UK

Corresponding author:

RA Wright, Cardiothoracic Division, The James Cook University

Hospital, Marton Road, Middlesbrough, TS4 3BW, UK.

Email: Robert.Wright@stees.nhs.uk 


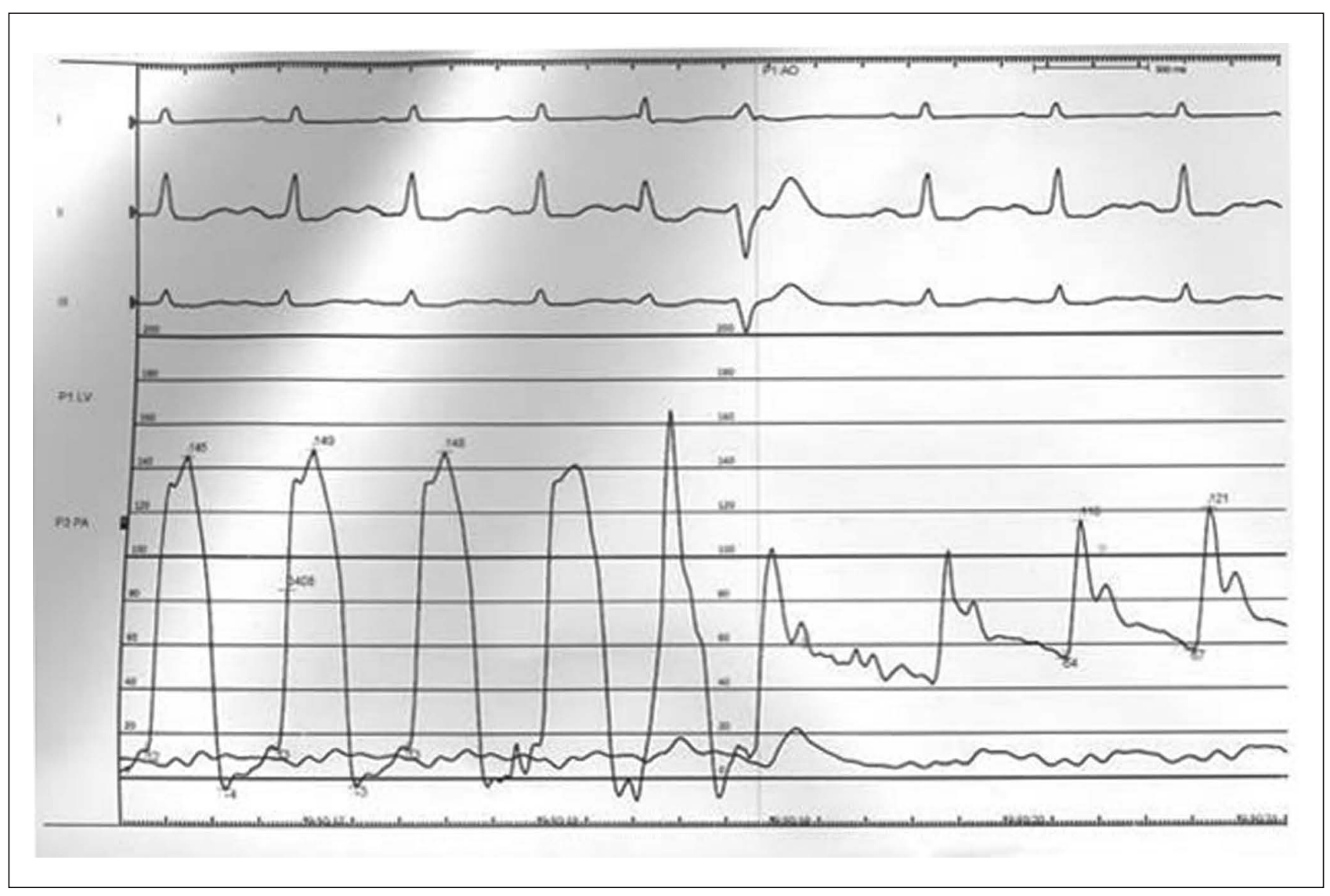

Figure I. Pressure trace on withdrawal from left ventricle into aorta.

His progress was complicated by the development of a loud pansystolic murmur associated with evidence of pulmonary oedema which was treated with intravenous furosemide and glyceryl trinitrate. At this point he had a sinus tachycardia of 110 beats per minute and blood pressure was maintained at $142 / 80 \mathrm{mmHg}$ The possibility of a ventricular septal defect or papillary muscle rupture was raised. The bedside echocardiogram showed turbulence in the LVOT and some evidence of mitral regurgitation but images were poor. He was taken back to the catheter laboratory where a right heart catheter did not show any evidence of an oxygen saturation step up. A left ventricular (LV) angiogram showed a dynamic LV cavity with only minor anterior wall hypokinesis and severe mitral regurgitation. The pressure trace obtained on withdrawal from the left ventricle into the aorta is shown in (Figure 1).

An intra-aortic balloon pump (IABP) was inserted. On 1:1 balloon augmentation his blood pressure fell as low as $70 / 46 \mathrm{mmHg}$. This was initially attributed to worsening mitral regurgitation. A subsequent transoesophageal echocardiogram showed a normal aortic valve and dynamic LVOT obstruction with systolic anterior motion of the anterior mitral leaflet against a prominent septal knuckle causing gross distortion of the valve and associated severe mitral regurgitation (Figure 2). The mid-interventricular septum measured $1.0 \mathrm{~cm}$ at end diastole (with a basal septal bulge of $1.4 \mathrm{~cm}$ ), and posterior wall $0.7 \mathrm{~cm}$. There was a dynamic LVOT gradient of $2.8 \mathrm{~m} / \mathrm{s}(31 \mathrm{mmHg})$ measured by continuous wave Doppler.

There was no evidence of chordal or papillary muscle rupture. The identification of the dynamic LVOT obstruction as a probable cause of the mitral regurgitation led to a change of treatment strategy. The intravenous nitrate therapy was stopped and the frequency of counterpulsation support was reduced. The patient was started on beta-blocker treatment (metoprolol $25 \mathrm{mg}$ t.d.s., subsequently changed to Bisoprolol $10 \mathrm{mg}$ o.d.) and given intravenous $0.9 \%$ saline fluid replacement. The IABP was stopped and removed. His blood pressure rose to $128 / 60 \mathrm{mmHg}$ and the murmur disappeared. He made good progress and a subsequent echocardiogram showed minimal LV impairment with mild aortic and mitral regurgitation. An angiotensin-converting enzyme inhibitor was avoided because of the possibility that it might exacerbate outflow tract obstruction.

\section{Discussion}

Dynamic LVOT obstruction with systolic anterior mitral leaflet motion in acute anterior myocardial infarction is a 


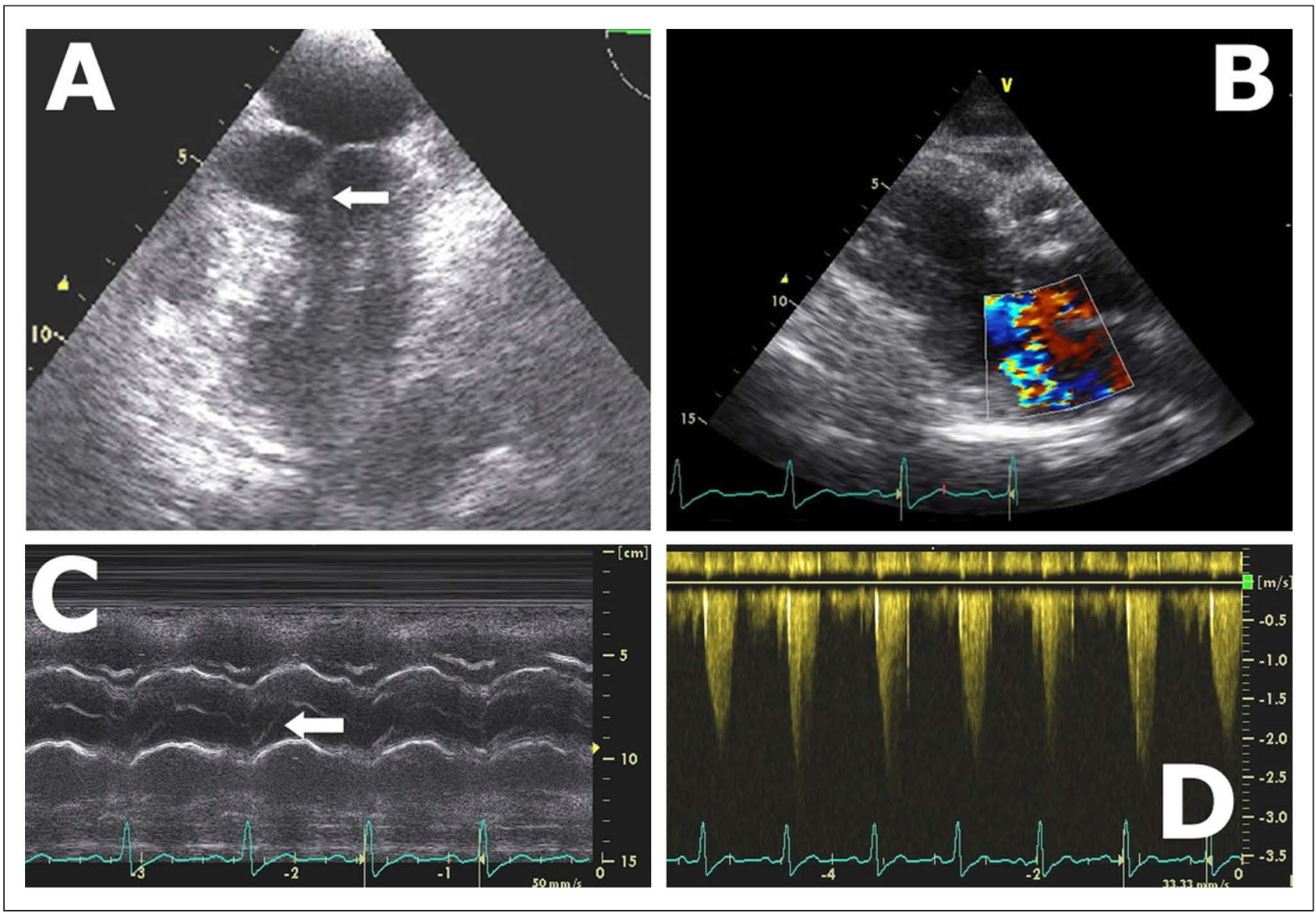

Figure 2. Transoesophageal echocardiography showing systolic anterior motion of the mitral valve leaflet (a, arrow) and transthoracic echocardiography showing severe mitral regurgitation (b). M-mode showing early closure of the aortic valve (c, arrow) and late peaking of the continuous-wave Doppler veocity curve from the left ventricular outflow tract (d).

potentially reversible complication that, in its most severe form, can result in cardiogenic shock. The distortion in left ventricular geometry causing outflow tract obstruction is thought to be due to increased contractility of the preserved myocardial segments. ${ }^{1-3}$

Clinically the complication is suggested by the development of either or both an ejection systolic murmur from the LVOT obstruction and pansystolic murmur of mitral regurgitation. It can be associated with low output state and pulmonary oedema.

The LVOT is positioned centrally in the heart. Below the level of the aortic valve, the outflow tract is bordered anteriorly and to the left by the infundibular septum. Posteriorly, it is bordered by the anterior leaflet of the mitral valve and the central fibrous body. The membranous septum is positioned anteriorly and to the right, beneath the junction of the right- and non-coronary cusps of the aortic valve. ${ }^{4}$ Congenital obstruction of the LVOT can be seen with subvalvar membranes or in the hypoplastic left heart syndrome. ${ }^{5}$ Dynamic left ventricular outflow tract obstruction is a pathogonomic observation in patients with hypertrophic obstructive cardiomyopathy. ${ }^{6}$ It has also been reported under other circumstances such as dobutamine administration, ${ }^{7}$ in stress-induced (takotsubo) cardiomyopathy, ${ }^{8}$ and in the postoperative period after mitral $^{9}$ or aortic ${ }^{10,11}$ valve surgery. Although stress-induced cardiomyopathy can mimic acute anterior myocardial infarction, it is typically associated with normal epicardial coronary arteries at angiography, or mild disease at most, whereas this patient had a focal stenosis of $74 \%$ diameter vessel obstruction measured by quantitative angiography in a vessel which appeared diffusely atheromatous. In the setting of acute myocardial infarction due to mid-left anterior descending artery obstruction, apical infarction with compensatory hypercontractility of the basal myocardial segments is postulated to decrease the size of the left ventricle outflow tract during systole. Accelerated blood flow across the narrow LVOT is thought to produce a Venturi effect with consequent displacement of the anterior mitral leaflet towards the septum, aggravating LVOT obstruction. This movement can result in the anterior mitral leaflet contacting the septum for a period of systole, effectively obstructing the outflow. The failure of the anterior mitral leaflet to coapt with the posterior leaflet in systole results in mitral regurgitation. ${ }^{1-3}$ 
Obstruction to the flow through the LVOT increases afterload and left ventricle wall stress. It is thought that in some cases with multivessel coronary disease, the development of dynamic LVOT obstruction by this mechanism does not occur due to inability of the basal segments to become hyperkinetic. In our patient, LVOT obstruction occurred in spite of a significant circumflex artery stenosis.

Consequences of LVOT obstruction in acute coronary syndrome include increased myocardial oxygen demand, expansion of the ischaemic zone, and hypotension. The increased systolic wall stress due to obstruction has been associated with cases of myocardial rupture. ${ }^{12}$

Management of dynamic LVOT obstruction is aimed at reversing the components of the postulated mechanism. Increasing LV filling by fluid administration, reducing contractility by the use of beta-blocker treatment or other negatively inotropic drugs and increasing afterload using an alpha-agonist have been used to improve the situation..$^{2,13,14}$ Inotropes, vasodilators, and an IABP should be avoided as they reduce the afterload and can worsen the haemodynamic condition. ${ }^{14,15}$ These were discontinued in our patient once the diagnosis was confirmed. Inotropes have been reported to induce transient systolic anterior mitral leaflet motion and LVOT obstruction in approximately $17-21 \%$ of otherwise healthy people. ${ }^{7,16}$ Calcium-channel blockers, especially verapamil, have been used where beta-blockers are contraindicated.

Dynamic LVOT obstruction should be considered as a potential mechanical complication of acute myocardial infarction in a patient with cardiogenic shock or hypotension, especially if these are refractory to or even made worse by the usual treatments. Clinical suspicion, early recognition, and appropriate management can significantly improve the outcome.

In our patient, our initial reaction was that he was likely to need emergency cardiac surgery. However, the identification of the LVOT obstruction as the cause of mitral regurgitation allowed us to treat him appropriately with fluids and beta-blocker therapy, rapidly improving his clinical status. We also discontinued the intravenous nitrate therapy and removed the IABP, both of which would have aggravated the degree of obstruction.

\section{References}

1. Haley JH, Sinak LJ, Tajik AJ, et al. Dynamic left ventricular outflow tract obstruction in acute coronary syndromes: an important cause of new systolic murmur and cardiogenic shock. Mayo Clinc Proc 1999; 74: 901-906.
2. Hrovatin E, Piazza R, Pavan D, et al. Dynamic left ventricular outflow tract obstruction in the setting of acute anterior myocardial infarction: a serious and potentially fatal complication? Echocardiography 2002; 19: 449-455.

3. Chockalingam A, Tejwani L, Aggarwal K, et al. Dynamic left ventricular outflow tract obstruction in acute myocardial infraction with shock: cause, effect and coincidence. Circulation 2007; 116: e110-e113.

4. Walmsley R. Anatomy of left ventricular outflow tract. Br Heart J 1979: 41: 263-267.

5. Hickey EJ, Caldarone CA and McCrindle BW. Left ventricular hypoplasia: a spectrum of disease involving the left ventricular outflow tract, aortic valve and aorta. JACC 2012; 59 Supplement: S43-S54.

6. Sherrid MV. Dynamic left ventricular outflow obstruction in hypertrophic cardiomyopathy revisited: significance, pathogenesis and treatment. Cardiol Rev 1998; 6: 135-145.

7. Pellikka PA, Oh JK, Bailey KR, et al. Dynamic intraventricular obstruction during dobutamine stress echocardiography. A new observation. Circulation 1992; 86: 1429-1432.

8. El Mahmoud R, Mansencal N, Pilliére R, et al. Prevalence and characteristics of left ventricular outflow tract obstruction in Tako-Tsubo syndrome. Am Heart J 2008; 156: 543-548.

9. Melero JM, Rodríguez I, Such M, et al. Left ventricular outflow tract obstruction with mitral mechanical prosthesis. Ann Thorac Surg 1999; 68: 255-257.

10. Bartunek J, Sys SU, Rodrigues AC, et al. Abnormal systolic intraventricular flow velocities after valve replacement for aortic stenosis. Mechanisms, predictive factors, and prognostic significance. Circulation 1996; 93: 712-719.

11. López Ayerbe J, Evangelista Masip A, Armada Romero $\mathrm{E}$, et al. Aparición de gradiente dinámico intraventricular después de la sustitución valvular aórtica en pacientes con estenosis aórtica severa. Rev Esp Cardiol 2002; 55: 127-134.

12. Bartunek J, Vanderheyden $M$ and de Bruyne. Dynamic left ventricle outflow tract obstruction after anterior myocardial infarction: a potential mechanism of myocardial rupture. Eur Heart $J$ 1995; 16: 1439-1442.

13. First International Study of Infarct Survival Collaborative Group. Randomized trial of intravenous atenolol among 16027 cases of suspected acute myocardial infarction. ISIS-1. Lancet 1986; 2: 57-66.

14. Sherrid MV, Pearle G and Gunsburg DZ. Mechanism of benefit of negative inotropes in obstructive hypertrophic cardiomyopathy. Circulation 1998; 97: 41-47.

15. Tse RW, Masindet S, Stavola T, et al. Acute myocardial infarction with dynamic outflow tract obstruction precipitated by intra-aortic balloon pump counterpulsation: Cathet Cardiovasc Diagn 1996; 39: 62-66.

16. Cohen R, Rivagorda J and Elhadad S. Asymmetrical septal hypertrophy complicated by dynamic left ventricular obstruction after intra-aortic balloon counterpulsation placement in the setting of anterior myocardial infarction. $J$ Invasive Cardiol 2006; 18: E207-E208. 